\title{
Epitope Mapping of Antidiacylglycerol Kinase $\alpha$ Monoclonal Antibody DaMab-2
}

\author{
Masato Sano, Mika K. Kaneko, and Yukinari Kato
}

Diacylglycerol kinase (DGK) is responsible for the enzymatic conversion of diacylglycerol (DG) to phosphatidic acid (PA). Both DG and PA serve as signaling molecules; therefore, DGK functions as a key enzyme between DG- and PA-mediated signaling. DGK $\alpha$, one of the $10 \mathrm{DGK}$ isozymes, is involved in T cell function and has been shown to localize in the cytoplasm and nucleus. Furthermore, DGK $\alpha$ translocates to the plasma membrane in response to $\mathrm{T}$ cell receptor stimulation. Recently, we developed a specific monoclonal antibody (mAb), DaMab-2 (mouse $\mathrm{IgG}_{1}$, kappa), against DGK $\alpha$. DaMab-2 is very useful in immunocytochemical analysis using HeLa cells. In this study, we characterized the binding epitope of DaMab-2 using Western blot and revealed that Cys246, Lys249, Pro252, and Cys253 of DGK $\alpha$ are important for DaMab-2 binding to the DGK $\alpha$ protein. Our findings can be applied for the production of more functional anti-DGK $\alpha \mathrm{mAbs}$.

Keywords: diacylglycerol kinase, DGK $\alpha$, monoclonal antibody, epitope mapping

\section{Introduction}

D IACYLGLYCEROL KINASE (DGK) phosphorylates diacylglycerol (DG) to produce phosphatidic acid (PA). ${ }^{(1,2)} \mathrm{DG}$ is a neutral lipid derived from various sources, including phosphatidylinositol 4,5-bisphosphate and phosphatidylcholine, and serves as a second messenger that activates the conventional and novel types of the protein kinase $\mathrm{C}$ (PKC) family, RasGRP, Unc-13, and canonical transient receptor potential channels. ${ }^{(2,3)} \mathrm{PA}$ acts as a messenger molecule that activates hypoxia-inducible factor- $1 \alpha$, atypical $\mathrm{PKC} \zeta$, and mammalian target of rapamycin. Therefore, DGK serves as a key enzyme between DG- and PA-mediated pathways.

In mammalian species, DGK constitutes an enzyme family comprising 10 isozymes. ${ }^{(1,2)}$ Each isozyme possesses a distinct molecular structure and subcellular localization pattern. $\mathrm{DGK} \alpha$ is the first identified enzyme of $80 \mathrm{kDa}$ that contains an EF-hand motif ( $\mathrm{Ca}^{2+}$-binding site), a $\mathrm{Zn}$ finger ( $\mathrm{C} 1$ domain, DG-binding site), and a catalytic domain. Various cell types and tissues express DGK $\alpha$ in normal cells, including glial cells, vascular cells, hepatocytes, adrenal medullary cells, fibroblasts, and lymphocytes, or in several cancer cells, such as melanomas and sarcomas. ${ }^{(3-11)}$ DGK $\alpha$ regulates cell proliferation in response to IL-2 stimulation in T cells ${ }^{(3)}$ and is involved in T cell receptor (TCR) signaling by modulating RasGRP activity. ${ }^{(12)} \mathrm{T}$ cells isolated from DGK $\alpha$-deficient mice show an altered activity of TCR signaling and hyperproliferation. ${ }^{(13)} \mathrm{DGK} \alpha$ localizes in the cytoplasm and translocates to the plasma membrane in response to external stimuli. $^{(14)}$

Because no specific anti-DGK $\alpha$ monoclonal antibody (mAb) was available to detect human DGK $\alpha$ by immunostaining, the localization of the native protein was not clarified. Recently, we reported a novel antihuman DGK $\alpha \mathrm{mAb}$, DaMab-2 (mouse $\operatorname{IgG}_{1}$, kappa), which is very useful in immunocytochemical analysis using HeLa cells. In this study, we characterized the binding epitope of DaMab-2 using Western blot.

\section{Materials and Methods}

\section{Plasmid preparation}

Human DGK $\alpha \mathrm{cDNA}^{(15)}$ was synthesized and subcloned into an expression vector, pMAL-c2 (New England Biolabs, Inc., Beverly, MA), with PA tag (GVAMPGAEDDVV), ${ }^{(16)}$ using the In-Fusion HD Cloning kit (Takara Bio, Inc., Shiga, Japan), named pMAL-c2-DGK $\alpha$-PA. The deletion mutants of $\mathrm{DGK} \alpha$ were produced by polymerase chain reaction (PCR) and subcloned into pMAL-c2 with PA tag using the InFusion HD Cloning kit. The substitution of DGK $\alpha$ amino acids 240-260 with either alanine or glycine in dC320 of DGK $\alpha$ was performed using the QuikChange Lightning SiteDirected Mutagenesis Kit (Agilent Technologies, Inc., Santa

Department of Antibody Drug Development, Tohoku University Graduate School of Medicine, Sendai, Japan. 
Clara, CA). These constructs were verified by direct DNA sequencing.

\section{Western blot analyses}

Competent Escherichia coli TOP-10 cells (Thermo Fisher Scientific, Inc., Waltham, MA) were transformed and cultured overnight at $37^{\circ} \mathrm{C}$ in Luria-Bertani (LB) medium (Thermo Fisher Scientific) containing $100 \mu \mathrm{g} / \mathrm{mL}$ ampicillin (FUJIFILM Wako Pure Chemical Corporation, Osaka, Japan). Cell pellets were resuspended in phosphate-buffered saline with $1 \%$ Triton X-100 and $50 \mu \mathrm{g} / \mathrm{mL}$ aprotinin (SigmaAldrich Corp.). Lysates were immunoprecipitated using amylose resin (New England Biolabs, Inc.) and boiled in sodium dodecyl sulfate sample buffer (Nacalai Tesque, Inc., Kyoto, Japan). The samples were electrophoresed on 5\%$20 \%$ polyacrylamide gels (FUJIFILM Wako Pure Chemical Corporation) and transferred onto a polyvinylidene difluoride membrane (Merck KGaA, Darmstadt, Germany). After blocking with $4 \%$ skim milk (Nacalai Tesque, Inc.) for $1 \mathrm{~h}$ at room temperature, the membrane was incubated with DaMab-2 ${ }^{(17)}$ overnight at $4^{\circ} \mathrm{C}$, NZ-1 (anti-PA tag) for $1 \mathrm{~h}$ at room temperature, or LpMab-13 (anti-PA tag) for $1 \mathrm{~h}$ at room temperature $^{(18)}$ and then with peroxidase-conjugated antimouse IgG (1:2000 dilution; Agilent Technologies, Inc.) or antirat IgG (1:10,000 dilution; Sigma-Aldrich Corp.) for $1 \mathrm{~h}$ at room temperature. The membrane was finally developed using ImmunoStar LD Chemiluminescence Reagent (FUJIFILM Wako Pure Chemical Corporation) using SayacaImager (DRC Co., Ltd., Tokyo, Japan).

\section{Results and Discussion}

Several anti-DGK $\alpha \mathrm{mAbs}$ are commercially available and have been reported to be useful in Western blot and immunocytochemical analyses. However, recombinant or overexpressed DGK $\alpha$ has been usually used in these studies.
A
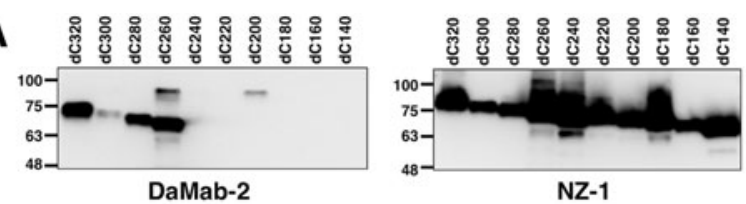

B

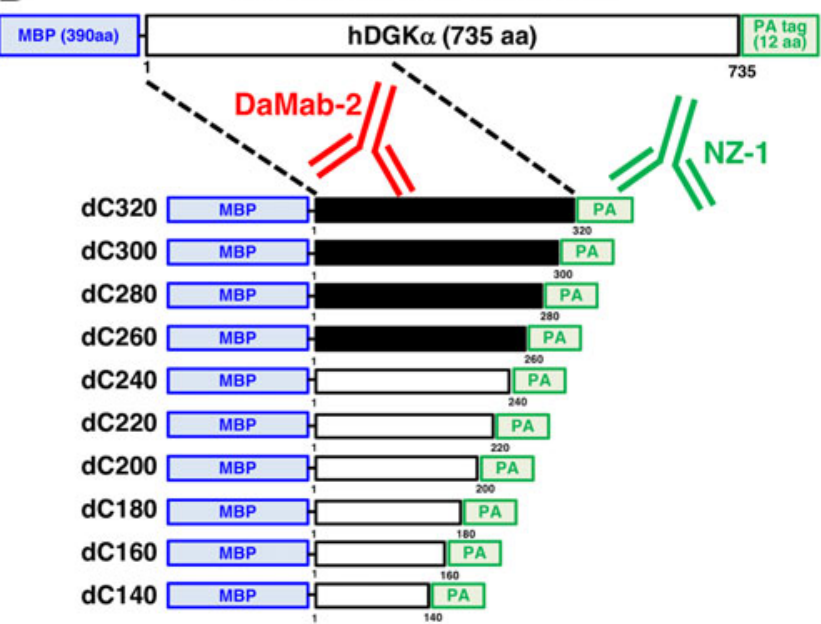

FIG. 1. Production of DGK $\alpha$ deletion mutants. (A) Immunoprecipitates of deletion mutants were electrophoresed and transferred onto PVDF membranes. After blocking, membranes were incubated with $20 \mu \mathrm{g} / \mathrm{mL}$ DaMab-2 or $1 \mu \mathrm{g} / \mathrm{mL}$ anti-PA tag (NZ-1) followed by peroxidaseconjugated antimouse or antirat IgG. (B) Schematic illustration of DaMab-2 epitope. Black bars, deletion mutants detected by DaMab-2; white bars, deletion mutants not detected by DaMab2 . hDGK $\alpha$, human diacylglycerol kinase $\alpha$; MBP, maltosebinding protein; PA, phosphatidic acid; PVDF, polyvinylidene difluoride.

Table 1. Epitope Mapping of DaMab-2 Using Point Mutants

\begin{tabular}{llll}
\hline Point mutants & \multicolumn{1}{c}{ Sequence } & DaMab-2 & LpMab-13 \\
\hline Y240A & ATVHDQCAMKALPCEVSTYAK & + & + \\
T241A & YAVHDQCAMKALPCEVSTYAK & + & + \\
V242A & YTAHDQCAMKALPCEVSTYAK & + & + \\
H243A & YTVADQCAMKALPCEVSTYAK & + & + \\
D244A & YTVHAQCAMKALPCEVSTYAK & + & + \\
Q245A & YTVHDACAMKALPCEVSTYAK & + & + \\
C246A & YTVHDQAAMKALPCEVSTYAK & + & + \\
A247G & YTVHDQCGMKALPCEVSTYAK & + \\
M248A & YTVHDQCAAKALPCEVSTYAK & + \\
K249A & YTVHDQCAMAALPCEVSTYAK & + & + \\
A250G & YTVHDQCAMKGLPCEVSTYAK & - \\
L251A & YTVHDQCAMKAAPCEVSTYAK & + \\
P252A & YTVHDQCAMKALACEVSTYAK & + \\
C253A & YTVHDQCAMKALPAEVSTYAK & + \\
E254A & YTVHDQCAMKALPCAVSTYAK & + \\
V255A & YTVHDQCAMKALPCEASTYAK & + \\
S256A & YTVHDQCAMKALPCEVATYAK & + \\
T257A & YTVHDQCAMKALPCEVSAYAK & + \\
Y258A & YTVHDQCAMKALPCEVSTAAK & + \\
A259G & YTVHDQCAMKALPCEVSTYGK & + \\
K260A & YTVHDQCAMKALPCEVSTYAA & + \\
\hline
\end{tabular}

Italic letters show the substitution of amino acid. 


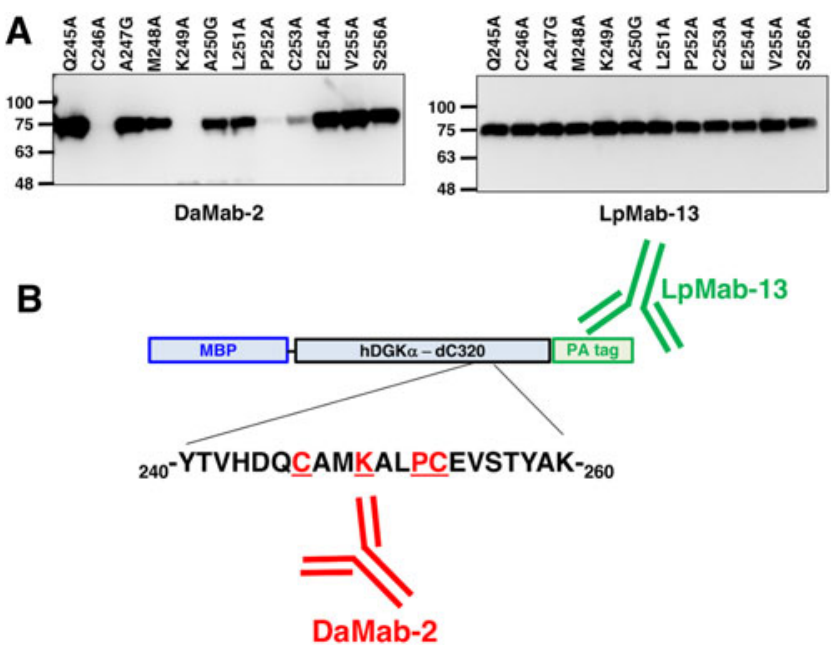

FIG. 2. Epitope mapping of DaMab-2 using deletion and point mutants of DGK $\alpha$. (A) Immunoprecipitates of point mutants were electrophoresed and transferred onto PVDF membranes. After blocking, membranes were incubated with $20 \mu \mathrm{g} / \mathrm{mL}$ DaMab-2 or $1 \mu \mathrm{g} / \mathrm{mL}$ anti-PA tag (LpMab13) followed by peroxidase-conjugated antimouse IgG. (B) Schematic illustration of DaMab-2 epitope. Red underlined letters, the critical amino acids for DaMab-2 recognition.

Recently, we immunized mice with recombinant DGK $\alpha$ and developed a clone (DaMab-2). ${ }^{(17)}$ DaMab-2 recognized only DGK $\alpha$ in enzyme-linked immunosorbent assay and did not react with other isozymes, such as DGK $\gamma, \mathrm{DGK} \zeta, \mathrm{DGK} \eta$, and DGK $\delta$. Furthermore, DaMab-2 is very useful in immunocytochemical analysis using HeLa cells.

As shown in Figure 1, we produced $10 \mathrm{C}$-terminal deletion mutants of $\mathrm{DGK} \alpha(\mathrm{dC} 320, \mathrm{dC} 300, \mathrm{dC} 280, \mathrm{dC} 260, \mathrm{dC} 240$, $\mathrm{dC} 220, \mathrm{dC} 200, \mathrm{dC} 180, \mathrm{dC} 160$, and dC140). Western blot demonstrated that DaMab-2 detected dC320, dC300, dC280, and $\mathrm{dC} 260$ but not dC240, dC220, dC200, dC180, dC160, and $\mathrm{dC140}$, although all deletion mutants were detected by an anti-PA tag mAb, NZ-1 (Fig. 1A), indicating that the Cterminus of the DaMab-2-epitope exists between amino acids 240 and 260. These results are summarized in Figure 1B.

We next produced the following $21 \mathrm{DGK} \alpha$ point mutants: Y240A, T241A, V242A, H243A, D244A, Q245A, C246A, A247G, M248A, K249A, A250G, L251A, P252A, C253A, E254A, V255A, S256A, T257A, Y258A, A259G, and K260A (Table 1). Western blot demonstrated that the anti-PA tag mAb, LpMab-13, detected Q245A, C246A, A247G, M248A, K249A, A250G, L251A, P252A, C253A, E254A, V255A, and S256A (Fig. 2A). In contrast, DaMab-2 strongly detected Q245A, A247G, M248A, A250G, L251A, E254A, V255A, and S256A, but did not detect mutants C246A and K249A and weakly detected P252A and C253A (Fig. 2A). Both DaMab-2 and LpMab-13 recognized the other point mutants, such as Y240A, T241A, V242A, H243A, D244A T257A, Y258A, A259G, and K260A (Table 1). DaMab-2 epitope is summarized in Figure 2B.

In conclusion, Cys246, Lys249, Pro252, and Cys253 are important for DaMab-2 binding to the DGK $\alpha$ protein. Our findings can be applied for the production of more functional anti-DGK $\alpha$ mAbs.

\section{Acknowledgments}

This research was supported, in part, by AMED under grant numbers JP18am0101078 (Y.K.), JP18am0301010 (Y.K.), and JP18ae0101028 (Y.K.), and by JSPS KAKENHI grant number 17K07299 (M.K.K.) and grant number 16K10748 (Y.K.).

\section{Author Disclosure Statement}

Y.K. received research funding from Ono Pharmaceutical Co., Ltd. The other authors have no conflict of interest.

\section{References}

1. Topham MK, and Epand RM: Mammalian diacylglycerol kinases: Molecular interactions and biological functions of selected isoforms. Biochim Biophys Acta 2009;1790:416424.

2. Goto K, Hozumi Y, Nakano T, Saino SS, and Kondo H: Cell biology and pathophysiology of the diacylglycerol kinase family: Morphological aspects in tissues and organs. Int Rev Cytol 2007;264:25-63.

3. Sakane F, Imai S, Kai M, Yasuda S, and Kanoh H: Diacylglycerol kinases: Why so many of them? Biochim Biophys Acta 2007;1771:793-806.

4. Goto K, Watanabe M, Kondo H, Yuasa H, Sakane F, and Kanoh H: Gene cloning, sequence, expression and in situ localization of $80 \mathrm{kDa}$ diacylglycerol kinase specific to oligodendrocyte of rat brain. Brain Res Mol Brain Res 1992; 16:75-87.

5. Yanagisawa K, Yasuda S, Kai M, Imai S, Yamada K, Yamashita T, Jimbow K, Kanoh H, and Sakane F: Diacylglycerol kinase alpha suppresses tumor necrosis factoralpha-induced apoptosis of human melanoma cells through NF-kappaB activation. Biochim Biophys Acta 2007;1771: 462-474.

6. Hasegawa H, Nakano T, Hozumi Y, Takagi M, Ogino T, Okada M, Iseki K, Kondo H, Watanabe M, Martelli AM, and Goto K: Diacylglycerol kinase zeta is associated with chromatin, but dissociates from condensed chromatin during mitotic phase in NIH3T3 cells. J Cell Biochem 2008; 105:756-765.

7. Nakano T, Hozumi Y, Goto K, and Wakabayashi I: Localization of diacylglycerol kinase epsilon on stress fibers in vascular smooth muscle cells. Cell Tissue Res 2009;337: 167-175.

8. Baldanzi G, Pietronave S, Locarno D, Merlin S, Porporato P, Chianale F, Filigheddu N, Cantelmo AR, Albini A, Graziani A, and Prat M: Diacylglycerol kinases are essential for hepatocyte growth factor-dependent proliferation and motility of Kaposi's sarcoma cells. Cancer Sci 2011; 102:1329-1336.

9. Nakano T, Hozumi Y, Goto K, and Wakabayashi I: Involvement of diacylglycerol kinase gamma in modulation of iNOS synthesis in Golgi apparatus of vascular endothelial cells. Naunyn Schmiedebergs Arch Pharmacol 2012; 385:787-795.

10. Nakano T, Hozumi Y, Iwazaki K, Okumoto K, Iseki K, Saito T, Kawata S, Wakabayashi I, and Goto K: Altered expression of diacylglycerol kinase isozymes in regenerating liver. J Histochem Cytochem 2012;60:130-138.

11. Hozumi Y, Akimoto R, Suzuki A, Otani K, Watanabe M, and Goto K: Expression and localization of the diacylglycerol kinase family and of phosphoinositide signaling 
molecules in adrenal gland. Cell Tissue Res 2015;362:295305.

12. Jones DR, D'Santos CS, Merida I, and Divecha N: T lymphocyte nuclear diacylglycerol is derived from both de novo synthesis and phosphoinositide hydrolysis. Int $\mathbf{J}$ Biochem Cell Biol 2002;34:158-168.

13. Olenchock BA, Guo R, Carpenter JH, Jordan M, Topham MK, Koretzky GA, and Zhong XP: Disruption of diacylglycerol metabolism impairs the induction of $\mathrm{T}$ cell anergy. Nat Immunol 2006;7:1174-1181.

14. Sanjuan MA, Jones DR, Izquierdo M, and Merida I: Role of diacylglycerol kinase alpha in the attenuation of receptor signaling. J Cell Biol 2001;153:207-220.

15. Schaap D, de Widt J, van der Wal J, Vandekerckhove J, van Damme J, Gussow D, Ploegh HL, van Blitterswijk WJ, and van der Bend RL: Purification, cDNA-cloning and expression of human diacylglycerol kinase. FEBS Lett 1990; 275:151-158.

16. Fujii Y, Kaneko M, Neyazaki M, Nogi T, Kato Y, and Takagi J: PA tag: A versatile protein tagging system using a super high affinity antibody against a dodecapeptide derived from human podoplanin. Protein Expr Purif 2014;95: 240-247.

17. Nakano T, Ogasawara S, Tanaka T, Hozumi Y, Mizuno S, Satoh E, Sakane F, Okada N, Taketomi A, Honma R, Na- kamura T, Saidoh N, Yanaka M, Itai S, Handa S, Chang YW, Yamada S, Kaneko MK, Kato Y, and Goto K: DaMab-2: Anti-human DGKalpha monoclonal antibody for immunocytochemistry. Monoclon Antib Immunodiagn Immunother 2017;36:181-184.

18. Ogasawara S, Kaneko MK, Honma R, Oki H, Fujii Y, Takagi M, Suzuki H, and Kato Y: Establishment of mouse monoclonal antibody LpMab-13 against human podoplanin. Monoclon Antib Immunodiagn Immunother 2016;35: 155-162.

Address correspondence to: Yukinari Kato Department of Antibody Drug Development Tohoku University Graduate School of Medicine New Industry Creation Hatchery Center Tohoku University 2-1, Seiryo-machi, Aoba-ku Sendai 980-8575 Japan

E-mail: yukinarikato@med.tohoku.ac.jp

Received: November 14, 2018 Accepted: December 10, 2018 